\title{
SAÚDE NA ESCOLA: O QUE É CERTO OU ERRADO QUANDO SE TRATA DE POSTURA?
}

\author{
Andressa Sartori Somekawa ${ }^{1}$ \\ Fernanda Flávia Cockel1 ${ }^{2}$ \\ Larissa Baraçal Bordon ${ }^{3}$ \\ Natália Novaes Pavani Araújo ${ }^{4}$ \\ Gabriel Tavares da Motta Esperança ${ }^{5}$ \\ Aline Suelen Pires ${ }^{6}$
}

\begin{abstract}
RESUMO: Este trabalho relata a experiência do Programa Saúde na Escola (PSE) com crianças de 7 a 11 anos de idade, as quais fazem parte do projeto de extensão "Fisioterapia Coletiva: ações no Morro Nova Cintra", localizado na cidade de Santos. O trabalho tem como objetivo construir, junto com os alunos envolvidos, práticas favoráveis à postura corporal, por meio de um novo conceito de saberes. Ou seja, as trocas de conhecimento e experiências interdisciplinares permitem a melhor apropriação da informação. Assim, o coletivo passa também a ser responsável por sua saúde, tendo a opção de evitar certas posturas, que adotadas por longos períodos, podem causar desconforto ou mesmo serem nocivas à saúde, além de identificar precocemente possíveis problemas posturais.
\end{abstract}

PALAVRAS-CHAVE: Crianças. Saúde Escolar. Postura.

\section{Health at school: what is right or wrong concerning good posture?}

\begin{abstract}
This paper reports the practical experience of the Health at School Program (Programa Saúde na Escola) for children from 7 to 11 years old, who are part of the extension project called "Collective Therapy: Actions in Nova Cintra Community" (Fisioterapia Coletiva: ações no Morro Nova Cintra), in the city of Santos, Brazil. The project aims to develop, with the students involved, practices that would favor a good body posture, using a new concept of knowledge. That is, exchanging knowledge and interdisciplinary experiences, that would allow students to better acquire information. Thus, the students become responsible for their own health. They can avoid postures that, when adopted for long periods, can cause discomfort and even be harmful to their health. It also allows an early identification of possible postural problems.
\end{abstract}

\footnotetext{
Graduanda em Fisioterapia pela Universidade Federal de São Paulo (Campus Baixada Santista), ex-bolsista PIBEX no projeto de extensão "Fisioterapia Coletiva: Ações no Morro Nova Cintra", membro do grupo de pesquisa "Trabalho, Saúde e Vulnerabilidade Social" da Universidade Federal de São Paulo e Universidade Federal de São Carlos (desomekawa@hotmail.com).

${ }^{2}$ Pós-doutora em Sociologia Universidade Federal de São Carlos, professora adjunta da Universidade Federal de São Paulo (Campus Baixada Santista), coordenadora do projeto de extensão "Fisioterapia Coletiva: Ações no Morro Nova Cintra" (fercockell@yahoo.com.br).

${ }^{3}$ Graduanda em Fisioterapia pela Universidade Federal de São Paulo (Campus Baixada Santista), ex-bolsista PIBEX no projeto de extensão "Fisioterapia Coletiva: Accões no Morro Nova Cintra", membro do grupo de pesquisa "Trabalho, Saúde e Vulnerabilidade Social" da Universidade Federal de São Paulo e Universidade Federal de São Carlos, bolsista de iniciação científica CNPq (lari.unifesp@gmail.com).

${ }^{4}$ Graduanda em Psicologia pela Universidade Federal de São Paulo (Campus Baixada Santista), ex-bolsista PIBEX no projeto de extensão "Fisioterapia Coletiva: Ações no Morro Nova Cintra", membro do grupo de pesquisa "Trabalho, Saúde e Vulnerabilidade Social" da Universidade Federal de São Paulo e Universidade Federal de São Carlos, monitora do eixo "Inserção Indivíduo na Sociedade" (natalia pavani@yahoo.com.br).

${ }^{5}$ Especialista em Fisioterapia Cardiorrespiratória pelo Instituto do Coração do Hospital de Clínicas da Faculdade de Medicina da Universidade de São Paulo, ex-bolsista PIBEX no projeto de extensão "Fisioterapia Coletiva: Ações no Morro Nova Cintra", membro do grupo de pesquisa "Trabalho, Saúde e Vulnerabilidade Social” da Universidade Federal de São Paulo e Universidade Federal de São Carlos (gabrieltava@ hotmail.com).

${ }^{6}$ Doutoranda em Sociologia pela Universidade Federal de São Carlos, bolsista FAPESP, membro do grupo de pesquisa "Trabalho, Saúde e

Vulnerabilidade Social" da Universidade Federal de São Paulo e Universidade Federal de São Carlos, colaboradora do projeto de extensão

"Fisioterapia Coletiva: Ações no Morro Nova Cintra" (aline.sociologia@gmail.com).
} 
KEYWORDS: Children. Health at school. Posture.

\title{
INTRODUÇÃO
}

\begin{abstract}
Sente direito! Fique direito! Mães, professores e outras pessoas dizem tantas vezes estas frases em boa fé e com a mais total confiança no que estão dizendo. Se lhes perguntasse como se faz para sentar ou ficar direito, responderiam: o que é que você quer dizer com isto? Você não sabe o que quer dizer direito? Direito é direito. (FELDENKRAIS, 1977, p. 91).
\end{abstract}

Pais e educadores bombardeiam diariamente a mente das crianças com estas frases. $\mathrm{O}$ mesmo ocorre com os profissionais de saúde que insistem em corrigir a postura das pessoas, de forma prescritiva e normativa, como se uma simples palavra de comando fosse determinar como interagirmos com o meio ao nosso redor.

Sem contar no caso dos trabalhadores, quando posturas, ditas corretas, são prescritas baseadas em um modelo reducionista do ser humano, estritamente biológico. Ao acreditar no "mito da postura correta" (ASSUNÇÃO, 2004, p. 42) é desconsiderado o fato de a postura adotada no ambiente de trabalho ser determinada pelo meio, pelas ferramentas utilizadas, pelos objetivos determinados, pelos resultados esperados e pelo próprio estado interno do trabalhador. A postura corporal é, portanto, "multideterminada e não fruto de uma casualidade ou de idiossincrasias pessoais" (ASSUNÇÃO, 2004, p. 53).

A postura, segundo sua própria definição, é uma disposição, ou melhor, uma posição, do corpo. Ou seja, uma atitude corporal para uma determinada atividade. Nos livros clássicos, trata-se da posição do corpo ou uma maneira de alguém sustentar seu próprio corpo (KISNER; COLBY, 2009). No entanto, a postura também pode ser descrita pelo alinhamento do corpo ou ainda, como uma orientação do mesmo em um ambiente qualquer (SHUMWAY-COOK, WOOLLACOTT, 2011).

A postura saudável é aquela em que a pessoa consegue manter-se com o mínimo de esforço muscular para estabelecer a sua própria forma de sustentação. Porém, por outro ângulo, a postura estereotipada pode aumentar a pressão sobre os elementos corporais e a sua distribuição sobre as estruturas tornam-se menos capazes de sustentá-lo, causando assim, possíveis alterações posturais, dores e lesões (ASSUNÇÃO, 2004).

No entanto, a postura ideal é realmente manter o corpo ereto? Para alguns profissionais da saúde, nem sempre essa afirmação é correta (ASSUNÇÃO, 2004; SHUMWAY-COOK, WOOLLACOTT, 2011). Existe um novo conceito sobre postura corporal que considera a flexibilidade (BRASIL, 2011) como ponto central, pois a alterações das posições e os alongamentos são alternativas para manter a coluna e o corpo saudáveis. Mas, para isto é preciso que o indivíduo perceba seu corpo, saiba dos seus limites e das possibilidades de protegê-lo.

O corpo não deixa de ser um esquema tridimensional que todos possuem de si mesmos, denominado 
imagem corporal (BARROS, 2005; SCHILDER, 1999). Entende-se por imagem corporal a figuração de nossos corpos formada em nossa mente, ou seja, o modo pelo qual o corpo apresentase para nós (SCHILDER, 1999), porém não é apenas uma construção cognitiva, mas também uma "reflexão dos desejos, atitudes emocionais e interação com os outros" (FISHER, 1999, p. 11).

Usualmente, os aspectos físicos parecem ser o foco quando se trata de postura, mas Schilder (1999, p. 101) afirma o contrário quando diz que "o modelo postural do corpo precisa ser construído, e é uma criação e uma construção, e não um dom. É a produção de uma forma, adquirido através de suas experiências". A postura é uma expressão do corpo que construímos, o qual pode ou não ser causado por algum distúrbio musculoesquelético, uma vez que existem diversas variáveis envolvidas na postura e não somente sua parte anatômica, biológica, transcendendo, portanto, a dualidade do indivíduo, de um lado, e da sociedade, de outro lado e à parte.

A esse respeito, Bourdieu (1983, p. 47) afirma existir "a interiorização da exterioridade e a exteriorização da interioridade". A partir do conceito de "habitus", demonstra que as relações humanas e os fenômenos sociais possuem sempre uma parte de reprodução, de "estrutural", daquilo que foge à ação consciente ou intencional dos sujeitos e outra dose de voluntarismo, de intencional, de mudança, de singularidade. O autor define "habitus" como:

[...] sistema de disposições duráveis, estruturas estruturadas predispostas a funcionarem como estruturas estruturantes, isto é, como princípio que gera e estrutura as práticas e as representações que podem ser objetivamente "regulamentadas" e "reguladas" sem que por isso sejam o produto de obediência de regras, objetivamente adaptadas a um fim, sem que se tenha necessidade da projeção consciente deste fim ou do domínio das operações para atingi-lo, mas sendo, ao mesmo tempo, coletivamente orquestradas sem serem o produto da ação organizadora de um maestro. (BOURDIEU, 1983 apud ORTIZ, 1994, p. 15).

Cabe destacar que, "habitus" não é o mesmo que hábito. A palavra hábito é definida como uma inclinação, sendo o comportamento que determinada pessoa aprende e repete frequentemente, sem pensar como deve executá-lo. A influência do "habitus" na postura introduz na discussão o lugar do agente social, do agente histórico. Ao relacionar o corpo e a saúde, Montagner(2006, p. 517)explica que

Bourdieu debita este trabalho de interiorização de uma hexis corporal à instância da educação primária, realizada primordialmente pela família e grupo social. Em seguida, essa educação primária passa pelo trabalho pedagógico, institucionalizado no sistema de ensino.

Tendo como referencial teórico o conceito de "habitus" e, portanto, uma visão ampliada de postura corporal, as ações realizadas por acadêmicos dos cursos de Fisioterapia e Psicologia da Universidade Federal de São Paulo (Unifesp) - Baixada Santista nas escolas da Nova Cintra têm por finalidade mostrar, por meio de atividades educativas e didáticas, que é possível às crianças obterem alternativas para proteger a estrutura corporal, sem a necessidade de imposição de normas ou prescrição de hábitos, ditos como "corretos" pelo modelo biomédico. Assim como preconizado pelo Programa Saúde na Escola (PSE) e por Bourdieu (1983), o ambiente escolar é 
um "espaço privilegiado para o desenvolvimento crítico e político, contribuindo na construção de valores pessoais, crenças, conceitos e maneiras de conhecer o mundo e interfere diretamente na produção social da saúde" (BRASIL, 2009, p. 96).

\section{MÉTODO}

Durante o ano de 2011, o projeto de extensão "Fisioterapia Coletiva: ações no Morro Nova Cintra", em parceria com a Unidade Básica de Saúde (UBS) local, desenvolveu diversas oficinas e ferramentas educacionais sobre postura corporal em uma das escolas municipais inseridas no território de abrangência, localizado em Santos-SP. Os resultados das vivências corporais vêm sendo analisados, após aprovação do Comitê de Ética/ Plataforma Brasil nº 32564/2012.

Participaram do projeto cerca de 200 crianças de 8 a 11 anos, em fase escolar. Inicialmente, os acadêmicos da Unifesp, juntamente com a coordenadora do projeto, elaboraram um roteiro das atividades para apresentá-las para as professoras da escola municipal, contudo manteve-se aberta a possibilidade de alteração das mesmas tanto pelas professoras, quanto pelos alunos.

Na etapa de planejamento, cada oficina foi elaborada a partir dos objetivos desejados, buscando desenvolver habilidades e as trocas contínuas de experiências entre todos os atores sociais envolvidos. Em comum, todas as atividades buscaram trabalhar o corpo em movimento e a emoção, em relação ao seu mundo interno e externo e suas interações e representações culturais e sociais:

a. Mímica corporal: a atividade consiste em fazer mímicas corporais no intuito de expressar sentimentos (dor, raiva, alegria e etc.) que influenciam a postura corporal.

b. Tipos de marcha: as crianças andam livremente pelo espaço e imitam diversos tipos de marcha (marcha de alguém com salto alto, de um soldado, carregando mochila etc.).

c. Percepção do desconforto: as crianças permanecem em posturas estáticas até sentirem qualquer forma de desconforto; assim que a sensação de desconforto for percebida, a criança é instruída a mudar de postura.

d. O toque: dinâmica realizada em duplas. Cada dupla toca os ombros e braços do colega, sentindo o delineamento muscular, proeminências ósseas etc. Durante este processo, as crianças serão instruídas a sentir por meio do toque.

e. Desenhando o próprio corpo: em uma folha em branco, as crianças devem desenhar seus próprios corpos. Durante a atividade, as crianças são instruídas a desenhar detalhes do corpo, se possível, órgãos, membros e características individuais, sem receberem dicas.

f. Relaxamento: sentir o próprio corpo, sua respiração, voltar à atenção para si. 
g. Desenho corporal coletivo: desenhar o contorno de uma das crianças no papel pardo ou no chão em giz e, em grupo, construir o corpo humano de acordo com a percepção das partes.

h. Amarrados e vendados: privados do uso do tato e da visão, as crianças têm seus pés amarrados, depois suas mãos e em seguida seus olhos vendados. Em cada etapa, o objetivo será manter uma bexiga no ar, não a deixando tocar o solo.

i. Conhecendo a coluna: consiste na apresentação de teatros sobre postura, jogo da memória das posturas mais penosas e menos prejudiciais, pôster com imagens corporais, apresentação palpável de protótipos de colunas - de massinha e caixas de fósforo - e um modelo de uma mão de madeira para ilustrar o funcionamento do membro.

j. Os sentimentos: selecionar figuras de revistas em que os sujeitos apresentem expressões faciais diversas. Propor que as crianças identifiquem quais as expressões faciais, o que as ocasionam e se elas próprias já vivenciaram situações semelhantes.

k. Interpretando os sentimentos: separados em grupos (meninos e meninas), cada criança vai à frente da sala e expressa um sentimento, verbalmente e corporalmente, relacionando-os com experiências vividas. As outras buscam descobrir o sentimento interpretado.

As atividades planejadas foram expostas e devidamente explicadas às crianças, buscando primeiramente extrair o máximo de conhecimento possível dos alunos para saber o que deveria ser acrescentado e esclarecido. Após cada ação, foi aberta uma roda de conversa no intuito de fomentar o aprendizado, entender as vivências e tirar dúvidas.

O conceito de postura empregado nas atividades não é meramente a condição anatômica, mas um conjunto de integração de toda a composição do corpo, uma vez que esse é o local do qual tudo acontece. $\mathrm{O}$ intuito não foi apenas levar para a classe o que é postura propriamente dita, mas mostrar que a postura é incorporada, em consequência do "habitus", dos costumes, do meio e do estado interno (historicamente determinados).

Para verificar como o conteúdo foi compreendido pelas crianças (capaz de ser retido e incorporado com o tempo no próprio corpo) sempre no encontro seguinte as crianças expunham o que haviam aprendido no encontro anterior.

\section{RESULTADO DAS ATIVIDADES}

A literatura recente mostra que um dos possíveis cenários de atuação da Fisioterapia Coletiva é a escola (BRASIL, 2009; FOLTRAN et al., 2012), permitindo identificar precocemente distúrbios cinesiofuncionais comuns na fase de desenvolvimento do escolar, evitando limitações funcionais e futuras sequelas crônico-degenerativas, decorrentes da sobrecarga das mochilas escolares, da postura sentada por longos períodos, bem como dos desvios posturais tardiamente identificados. 
A orientação postural é apontada como mais eficiente na idade escolar (BRASIL, 2009; REBOLHO; CASAROTTO; JOÃO, 2009), pois as crianças jovens não conhecem os maus hábitos, e as ações de saúde de prevenção de nível secundário têm proporcionado resultados positivos quanto à diminuição das incapacidades. Estratégias educativas voltadas para esta faixa etária - história em quadrinhos, jogos de memória, quebra-cabeças, desenhos - incitam as crianças dando ensejo a um conhecer e cuidar do próprio corpo, valorizando e adotando hábitos saudáveis como uns dos aspectos básicos da qualidade de vida, com isso, adquirem mais responsabilidade em relação a sua saúde e a saúde coletiva (REBOLHO; CASAROTTO; JOÃO, 2009), bem como as ajudará a encontrar soluções locais e individuais para os riscos ergonômicos (FOLTRAN et al., 2012).

Entretanto, a maior parte dos estudos sobre a atuação da Fisioterapia em ambiente escolar limita-se a pesquisas sobre avaliação postural, fatores de risco ou, no máximo, sobre orientações prescritivas sobre posturas tidas como corretas, apenas considerando os aspectos biomecânicos ou medidas antropométricas das crianças, bem como o peso de suas mochilas (LEMOS; SANTOS; GAYA, 2012; SOUZA JUNIOR et al., 2011).

O projeto de extensão da Unifesp no Morro Nova Cintra é singular, pois a proposta é atuar exclusivamente na atenção básica, por meio da promoção da saúde e da prevenção primária, tendo o "habitus" como conceito norteador. O intuito é mostrar para as crianças o quanto é importante conhecer o seu corpo, seus limites, seus desconfortos e formas de protegê-lo, evitando posturas assimétricas que, quando mantidas por muito tempo, podem se tornar nocivas. Buscou-se não forçá-las a fazerem nada contra a sua vontade, nem impor uma divisão de cargos, do qual o aluno aprende e os profissionais da saúde ensinam, pelo contrário cada encontro foi uma construção mútua, bidirecional, com trocas de saberes e experiências.

As oficinas trouxeram um resultado favorável, pois as crianças somaram o que foi ensinado com o conhecimento que possuíam, passando adiante o que apreenderam e principalmente, incorporando atitudes e/ou comportamentos que passam pela lógica da retenção conforme o conceito elástico de "habitus", permitindo a

[...] absorção de formas corporais e de posturas, que, em longo prazo, acabam por tornar-se um sistema operatório, um sistema visível de conhecimento e reconhecimento, uma substância, com qualidades sensíveis e explicitáveis, capazes de dar uma visão de conjunto do indivíduo ou de um grupo de indivíduos. Essa sedimentação pressupõe a ação no tempo, traz implícita a história. Pressupõe a incorporação, o tornar-se corpo. Essa hexis é composta de um capital físico ou corporal, correspondente a uma disposição e a uma trajetória individual, mas também de uma dimensão coletivizada, de grupo. Esse poder de retenção é um poder basicamente corporal, ainda que não se conheçam os mecanismos dessa capacidade de memorização física. (MONTAGNER, 2006, p. 517).

Na Figura 1 são apresentadas as categorizações das atividades realizadas (BORDON, 2012), de acordo com a legenda abaixo dividida em subcategorias: 
Figura 1 - Representação visual das atividades realizadas (em cinza os aspectos presentes).

\begin{tabular}{|c|c|c|c|c|c|c|c|c|c|c|c|c|}
\hline CATEC & RIAS & 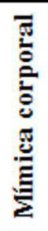 & 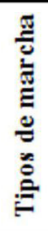 & 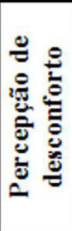 & $\frac{\stackrel{\Xi}{E}}{\stackrel{\Xi}{0}}$ & 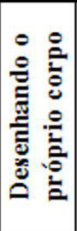 & 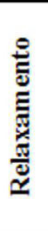 & 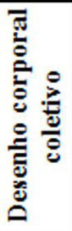 & 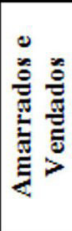 & 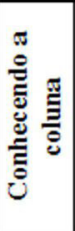 & 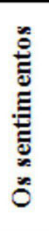 & 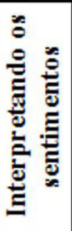 \\
\hline$\cong$ & la & & & & & & & & & & & \\
\hline$\stackrel{\frac{\pi}{3}}{3}$ & lb & & & & & & & & & & & \\
\hline$\stackrel{\frac{\nu}{g}}{g}$ & lc & & & & & & & & & & & \\
\hline$\overline{\underline{0}}$ & ld & & & & & & & & & & & \\
\hline$\approx$ & le & & & & & & & & & & & \\
\hline & $2 a$ & & & & & & & & & & & \\
\hline$\stackrel{\pi}{\circ}$ & $2 \mathrm{~b}$ & & & & & & & & & & & \\
\hline 巳 & $2 \mathrm{c}$ & & & & & & & & & & & \\
\hline 前哭 & $2 \mathrm{~d}$ & & & & & & & & & & & \\
\hline 8 & $2 e$ & & & & & & & & & & & \\
\hline & $2 f$ & & & & & & & & & & & \\
\hline & $3 a$ & & & & & & & & & & & \\
\hline$\overline{\underline{\pi}}$ & $3 \mathrm{~b}$ & & & & & & & & & & & \\
\hline 袍 & $3 c$ & & & & & & & & & & & \\
\hline 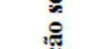 & $3 \mathrm{~d}$ & & & & & & & & & & & \\
\hline 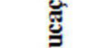 & $3 e$ & & & & & & & & & & & \\
\hline 胥 & $3 \mathrm{f}$ & & & & & & & & & & & \\
\hline & $3 \mathrm{~g}$ & & & & & & & & & & & \\
\hline . & $4 a$ & & & & & & & & & & & \\
\hline 氖 & $4 \mathrm{~b}$ & & & & & & & & & & & \\
\hline & $4 c$ & & & & & & & & & & & \\
\hline & $5 \mathbf{a}$ & & & & & & & & & & & \\
\hline 音 & $5 b$ & & & & & & & & & & & \\
\hline$\ddot{\mathscr{n}}$ & $5 \mathrm{c}$ & & & & & & & & & & & \\
\hline & $5 d$ & & & & & & & & & & & \\
\hline
\end{tabular}

Fonte: Bordon (2012).

Legenda: 1) Psicomotricidade: 1a - perceber o corpo de maneira global. 1b unir as diversas partes do corpo realizando uma montagem da figura humana; 1c - identificar cada parte do corpo e seus detalhes; 1d - conscientizar-se de que o corpo é formado de diversas partes e de que cada uma tem detalhes específicos; $1 \mathrm{e}$ - relacionar as partes do corpo ou detalhes com suas respectivas funções. 2) Comunicação-expressão: $2 \mathrm{a}$ - identificar os próprios sentimentos; $2 \mathrm{~b}$ - relacionar sentimentos e expressão corporal; $2 \mathrm{c}$ - expressar os próprios sentimentos (verbalmente, corporalmente ou graficamente); $2 \mathrm{~d}$ - ouvir o outro com atenção; $2 \mathrm{e}$ - explorar o próprio universo pessoal; $2 \mathrm{f}$ - relacionar sentimentos e experiências vividas. 3) Educação Sensorial: 3a - comparar estímulos observando suas semelhanças e diferenças; $3 b$ - desenvolver a atenção concentrada sobre dados visuais; $3 \mathrm{c}$ - coordenar a visão com movimentos do corpo; $3 \mathrm{~d}$ - demonstrar concentração da atenção em determinados estímulos, ignorando os irrelevantes; $3 \mathrm{e}$ - memorizar características de estímulos apresentados; $3 \mathrm{f}$ - identificar direções: para cima, para baixo, à direita, à esquerda; $3 g$ - observar, apalpar e perceber os estímulos pelo tato. 4) Iniciação à ciência: $4 \mathrm{a}$ - perceber-se como ser vivo; $4 \mathrm{~b}$ - distinguir seres vivos; $4 \mathrm{c}-$ distinguir objetos. 5) Sociais: 5a - interação em grupo; 5b-autonomia; 5c-lidar com as diferenças; $5 \mathrm{~d}$ - identificar em relação ao grupo família. 


\section{CONSIDERAÇÕES FINAIS}

O projeto de extensão conseguiu promover, ao mesmo tempo, conhecimento e autonomia, competências essenciais para a melhoria da qualidade de vida dos atores sociais envolvidos. A escola mostrou-se um cenário rico para as práticas promotoras da saúde, preventivas e de educação para saúde promovidas no território da UBS (BRASIL, 2009), assim como para a ampliação do campo de atuação dos profissionais da saúde, de maneira interdisciplinar, intersetorial, voltados para a atenção básica e focados nas reais demandas sociais (SHUMWAYCOOK; WOOLLACOTT, 2011; COCKELL; TELLINI, 2011; BISPO JÚNIOR, 2010).

Os resultados das oficinas mostraram que os problemas da postura não se resolvem apenas pela educação postural, ou prescrição de normas, cabendo aos profissionais de saúde "empoderar" os sujeitos, desde a tenra infância para perceberem o próprio corpo, seus limites físicos, desconfortos causados pela manutenção prolongada de uma mesma postura, bem como aprender as melhores formas para proteger sua coluna.

Foi possível ensiná-los que existem posturas favoráveis à saúde e que, mesmo estas posturas mais neutras, se mantidas por longos períodos, como acontece com a postura sentada na escola, são nocivas caso não possam ser alteradas. $O$ impacto social da extensão foi verificado por meio do envolvimento das crianças e dos professores com o novo conceito de aprendizado implantado, além de torná-los multiplicadores na família e na comunidade sobre os temas abordados.

Por meio das vivências corporais apresentadas, considerando o lugar do agente social, as crianças aprendem porque se interessam, porque passam conhecer e a reconhecer tais formas corporais, ao mesmo tempo em que interagem com o grupo, sentindo não somente o seu corpo, mas também os dos colegas, sem estabelecer o que é certo e o errado. Ou seja, levam consigo a definição mais rara sobre postura, aquela a qual muitos ainda não conhecem: a da postura como determinada pela atitude corporal que colocamos no corpo, historicamente influenciada e socialmente determinada.

\section{REFERÊNCIAS}

ASSUNÇÃO, A. A cadeirologia e o mito da postura correta. Revista Brasileira de Saúde Ocupacional, São Paulo, v. 29, n. 110, p. 41-55, 2004.

BARROS, D. D. Imagem corporal: a descoberta de si mesmo. História Ciência e SaúdeManguinhos, Rio de Janeiro, v. 12, n. 2, p. 547-554, ago./out. 2005.

BISPO JÚNIOR, J. P. Fisioterapia e saúde coletiva: desafios e novas responsabilidades profissionais. Ciência \& Saúde Coletiva, Rio de Janeiro, v.15, suppl. 1, p. 1627-1636, 2010.

BORDON, L. B. Fisioterapia coletiva na escola: contribuições e práticas pedagógicas. Relatório Parcial da Iniciação Científica-CNPq, 2012. 
BOURDIEU, P. Algumas propriedades dos campos. In: BOURDIEU, P. Questões de Sociologia. Rio de Janeiro: Marco Zero, 1983.

BRASIL. Ministério da Saúde. Portal da Saúde. Postura ideal: novo conceito aponta para flexibilidade corporal e emocional. 2011. Disponível em: < http://www.webradio.saude.gov.br/ noticia.php?codigo_noticia=PDMS111177>. Acesso em: 13 out. 2012.

BRASIL. Secretaria de Atenção à Saúde. Caderno de atenção básica na escola. Departamento de Atenção Básica. Brasília, DF: Ministério da Saúde, 2009.

COCKELL, F. F.; TELLINI, G. G. Fisioterapia coletiva no Morro Nova Contra: rompendo com saberes e fazeres tradicionais. In: SIMPÓSIO DE SAÚDE COLETIVA E SAÚDE MENTAL, 5., Belo Horizonte. Anais... Belo Horizonte: UFMG, 2011.

FELDENKRAIS, M. Consciência pelo movimento. São Paulo: Summus; 1977.

FISHER, S. The evolution of psychological concepts about the body. In: CASH, T. F.; PRUZINSKY, T. (Org.). Body images: development, deviance and change. New York: The Guilford Press; 1999.

FOLTRAN, F. A. et al. Effects of an educational back care program on Brazilian schoolchildren's knowledge regarding back pain prevention. Revista Brasileira de Fisioterapia, São Carlos, v.16, n. 2, p. 128-133, mar./abr. 2012.

KISNER, C.; COLBY, L. A. Exercícios terapêuticos: fundamentos e técnicas. 5. ed. São Paulo: Manole, 2009.

LEMOS, A. T. de; SANTOS, F. R. dos; GAYA, A. C. A. Hiperlordose lombar em crianças e adolescentes de uma escola privada no sul do Brasil: ocorrência e fatores associados. Cadernos de Saúde Pública, Rio de Janeiro, v. 28, n. 4, p. 781-788, abr. 2012.

MONTAGNER, M. A. Pierre Bourdieu, o corpo e a saúde: algumas possibilidades teóricas. Ciência \& Saúde Coletiva, Rio de Janeiro, v. 11, n. 2, p. 515-526, jun./out. 2006.

ORTIZ, R. À procura de uma sociologia da prática. In: ORTIZ, R. (Org.). Pierre Bourdieu: sociologia. 2. ed. São Paulo: Ática,1994.

REBOLHO, M. C. T.; CASAROTTO, R. A.; JOÃO, S. M. A. Estratégias para ensino de hábitos posturais em crianças: história em quadrinhos versus experiência prática. Fisioterapia e Pesquisa, São Paulo, v.16, n.1, p. 46-51, jan./mar. 2009.

SCHILDER, Paul. A imagem do corpo: as energias construtivas da psiquê. São Paulo: Martins Fontes, 1999.

SHUMWAY-COOK, A.; WOOLLACOTT, M. H. Motor control: translating research into clinical Practice. Philadelphia: Lippincott Williams \& Wilkins, 2011. 
SOUZA JUNIOR, J. V. et al. Perfil dos desvios posturais da coluna vertebral em adolescentes de escolas públicas do município de Juazeiro do Norte-CE. Fisioterapia e Pesquisa, São Paulo, v. 18. n. 4, p.311-316, out./dez. 2011.

Submetido em 23 de novembro de 2012.

Aprovado em 17 de dezembro de 2012. 